Research Article

\title{
Related Fixed Point Theorems via General Approach of Simulations Functions
}

\author{
Kastriot Zoto (iD), ${ }^{1}$ Nabil Mlaiki $\mathbb{D}^{2},{ }^{2}$ and Hassen Aydi $\mathbb{D i}^{3,4,5}$ \\ ${ }^{1}$ Department of Mathematics and Computer Sciences, Faculty of Natural Sciences, University of Gjirokastra, Gjirokastra, Albania \\ ${ }^{2}$ Department of Mathematics and General Sciences, Prince Sultan University, P. O. Box 66833, Riyadh 11586, Saudi Arabia \\ ${ }^{3}$ Nonlinear Analysis Research Group, Ton Duc Thang University, Ho Chi Minh City, Vietnam \\ ${ }^{4}$ Faculty of Mathematics and Statistics, Ton Duc Thang University, Ho Chi Minh City, Vietnam \\ ${ }^{5}$ China Medical University Hospital, China Medical University, Taichung 40402, Taiwan
}

Correspondence should be addressed to Hassen Aydi; hassen.aydi@tdtu.edu.vn

Received 29 May 2020; Accepted 31 July 2020; Published 4 September 2020

Academic Editor: Alfred Peris

Copyright ( $) 2020$ Kastriot Zoto et al. This is an open access article distributed under the Creative Commons Attribution License, which permits unrestricted use, distribution, and reproduction in any medium, provided the original work is properly cited.

In this work, we extend and complement some results in view of general and wider structures, such as $b$-metric spaces. By considering existing classes of $\mathrm{Z}$-contractions and $\Psi$-simulating functions with a solid impact in database results of fixed point theory, we introduce a new general class of simulating functions, called as $\Psi-s$ simulation functions, and also types of $\kappa_{\psi-s}-$ contractions in a more general framework. This approach covers, extends, and unifies several published works in the early and late literature.

\section{Introduction}

Some of the significant generalizations of metric fixed point theory are related with the well-known Banach Contraction Principle [1] and classical contractions such as Boyd and Wong, Geraghty, Browder, and Ciric. In recent years, the theory of fixed points has attracted widespread attention and has been rapidly growing. It was massively studied by many researchers giving new results by using classes of implicit functions defining new and large contractive conditions. Recently, Khojasteh et al. [2] presented the notion of $\mathrm{Z}$-contractions involving a new class of simulation functions that has been used and improved by many authors in various spaces, see [3-30]. Authors in [19] proposed new notion $\Psi$-simulation functions and established the type of $\mathrm{Z}_{\psi}$-contractions.

Inspired by the above works, in this paper we introduce a new class of general type of $\Psi-s$ simulation functions, defined in the setting of $b$-metric-like spaces. This class generalizes further and complements some results given in the framework of $b$-metric spaces.

\section{Preliminaries}

Definition 1 (see [6]). Let $X$ be a nonempty set and $s \geq 1$ be a given real number. A mapping $d: X \times X \longrightarrow[0,+\infty)$ is called a $b$-metric-like if for all $x, y, z \in X$, the following conditions are satisfied:

$$
\begin{aligned}
& \mathrm{d}(x, y)=0 \text { implies } x=y, \\
& \mathrm{~d}(x, y)=\mathrm{d}(y, x), \\
& \mathrm{d}(x, y) \leq s[\mathrm{~d}(x, z)+\mathrm{d}(z, y)] .
\end{aligned}
$$

The pair $(X, d)$ is called a $b$-metric-like space.

In a $b$-metric-like space $(X, d)$, if $x, y \in X$, and $\mathrm{d}(x, y)=0$, then $x=y$; however, the converse need not be true, and $\mathrm{d}(x, x)$ may be positive for $x \in X$.

Definition 2 (see [6]). Let $(X, d)$ be a $b$-metric-like space with parameter $s \geq 1$ and let $\left\{x_{q}\right\}$ be any sequence in $X$ and $x \in X$. Then, we have the following: 
(a) $\left\{x_{q}\right\}$ is said to be convergent to $x$ if $\lim _{q \longrightarrow+\infty} \mathrm{d}\left(x_{q}, x\right)=\mathrm{d}(x, x)$

(b) $\left\{x_{q}\right\}$ is said to be a Cauchy sequence in $(X, d)$ if $\lim _{q, p \longrightarrow+\infty} \mathrm{d}\left(x_{q}, x_{p}\right)$ exists and is finite

(c) The pair $(X, d)$ is called a complete $b$-metric-like space if, for every Cauchy sequence $\left\{x_{q}\right\}$ in $X$, there is $x \in X$ such that $\lim _{q, p \longrightarrow+\infty} \mathrm{d}\left(x_{q}, x_{p}\right)=\lim _{q \longrightarrow+\infty}$ $\mathrm{d}\left(x_{q}, x\right)=\mathrm{d}(x, x)$

Lemma 1 (see $[6,29,30])$. Let $\left\{x_{q}\right\}$ and $\left\{y_{q}\right\}$ be two sequences in $(X, d)$ that converge to $x$ and $y$, respectively. Then, we have

$$
s^{-2} \mathrm{~d}(x, y)-s^{-1} \mathrm{~d}(x, x)-\mathrm{d}(y, y) \leq \liminf _{q \longrightarrow+\infty} \mathrm{d}\left(x_{q}, y_{q}\right) \leq \limsup _{q \longrightarrow+\infty} \mathrm{d}\left(x_{q}, y_{q}\right) \leq s \mathrm{~d}(x, x)+s^{2} \mathrm{~d}(y, y)+s^{2} \mathrm{~d}(x, y) .
$$

In particular, $\mathrm{d}(x, y)=0 \Longrightarrow \lim _{q \longrightarrow+\infty} \mathrm{d}\left(x_{q}, y_{q}\right)=0$.

Also, for each $z \in X$, the above inequality becomes

$$
s^{-1} \mathrm{~d}(x, z)-\mathrm{d}(x, x) \leq \liminf _{q \longrightarrow+\infty} \mathrm{d}\left(x_{q}, z\right) \leq \limsup _{q \longrightarrow+\infty} \mathrm{d}\left(x_{q}, z\right) \leq s \mathrm{~d}(x, z)+s \mathrm{~d}(x, x) .
$$

In particular, if, $\mathrm{d}(x, x)=0$, then

$$
s^{-1} \mathrm{~d}(x, z) \leq \liminf _{q \longrightarrow+\infty} d\left(x_{q}, z\right) \leq \limsup _{q \longrightarrow+\infty} \mathrm{d}\left(x_{q}, z\right) \leq s \mathrm{~d}(x, z)
$$

Lemma 2 (see [23]). Let $\left\{x_{q}\right\}$ be a sequence in theb-metriclike space $(X, d)$ with parameter $s \geq 1$, such that

$$
\lim _{q \longrightarrow+\infty} \mathrm{d}\left(x_{q}, x_{q+1}\right)=0 \text {. }
$$

If $\lim _{q, p \longrightarrow+\infty} \mathrm{d}\left(x_{q}, x_{p}\right) \neq 0$, then there are $\varepsilon>0$ and two sequences of natural numbers $p(k), q(k)$ with $q_{k}>p_{k}>k$, (positive integers) such that

$$
\begin{gathered}
\mathrm{d}\left(x_{p_{k}}, x_{q_{k}}\right) \geq \varepsilon, \\
\mathrm{d}\left(x_{p_{k}}, x_{q_{k}-1}\right)<\varepsilon, \\
\frac{\varepsilon}{s^{2}} \leq \limsup _{k \longrightarrow \infty} \mathrm{d}\left(x_{p_{k}-1}, x_{q_{k}-1}\right) \leq \varepsilon s, \\
\frac{\varepsilon}{s} \leq \limsup _{k \longrightarrow \infty} \mathrm{d}\left(x_{q_{k}-1}, x_{p_{k}}\right) \leq \varepsilon, \frac{\varepsilon}{s} \leq \limsup _{k \longrightarrow \infty} \mathrm{d}\left(x_{p_{k}-1}, x_{q_{k}}\right) \leq \varepsilon s^{2} .
\end{gathered}
$$

Note: in the continuous section of the paper, we will use $\left(X_{*}, d, s\right)$ (resp. $\left.(X, d, s)\right)$ to denote that the space with parameter $s \geq 1$ is complete (resp. noncomplete).

\section{Main Results}

Let $\left(X_{*}, d, s\right)$ be a $b$-metric-like space and $\Psi([0,+\infty))$ represent the collection of continuous functions $\psi:[0,+\infty) \longrightarrow[0,+\infty)$ with the following properties: $\left(\psi_{1}\right): \psi$ is strictly increasing,

$\left(\psi_{2}\right): \psi(m)=0, \quad$ iff $m=0$.

Definition 3. A function $\kappa:[0,+\infty)^{2} \longrightarrow R$ is a $\Psi-s$ simulation function if there are $\psi \in \Psi$ and a coefficient $\lambda \geq 1$ so that

$\left(\kappa_{1}\right): \kappa(t, v)<\psi(v)-\psi\left(s^{\lambda} t\right)$ for all $t, v>0$

$\left(\kappa_{2}\right)$ : If $\left\{t_{n}\right\},\left\{v_{n}\right\}$ are sequences in $(0, \infty)$ such that $\lim _{n \longrightarrow+\infty} t_{n}=\lim _{n \longrightarrow+\infty} v_{n}=l>0$, and $t_{n} \leq v_{n}$, then $\lim _{n \longrightarrow+\infty} \sup \kappa\left(t_{n}, v_{n}\right)<0$

Remark 1

If in the definition above we take $s=1$, then we obtain the definition of a $\Psi$ - simulation function.

If we take $\psi$ as the identity function, then we get a definition of an $s$ - simulation function.

If we take $s=1$ and $\psi(v)=v$, then we get the definition of a simulation function.

We denote by $K_{\psi-s}$ the set of all $\Psi-s$ simulation functions. In the following example, we give such a kind of functions.

Example 1. Let $\kappa:[0,+\infty)^{2} \longrightarrow R$ be defined by

(1) $\kappa(t, v)=c \psi(v)-\psi(s t)$ for all $t, v \in(0,+\infty)$, where $c \in(0,1)$

(2) $\kappa(t, v)=\psi(v)-\phi(v)-\psi\left(s^{\lambda} t\right)$ for all $t, v \in(0,+\infty)$, where $\phi:[0,+\infty) \longrightarrow[0,+\infty)$ is such that $\liminf _{t \longrightarrow v} \phi(t)>0$ for all $v>0$ 
(3) $\kappa(t, v)=\phi(v)-\psi\left(s^{\lambda} t\right)$ for all $t, v \in(0,+\infty)$, where $\psi ; \phi:[0,+\infty) \longrightarrow[0,+\infty)$ are continuous and $\psi$ is increasing such that $\phi(v)<\psi(v)$ for all $v>0$

(4) $\kappa(t, v)=F(\psi(v), \varphi(v))-\psi\left(s^{\lambda} t\right)$ for all $t, v \in$ $(0,+\infty)$, where $F: R^{+} \times R^{+} \longrightarrow R$ is a C-class function where $F$ is continuous such that $F(t, v)<t$ for all $t>0$

For a self-mapping $f: X \longrightarrow X$, we denote by $A(x, y)$ the following:

$$
A(x, y)=\max \left\{\mathrm{d}(x, y), \mathrm{d}(x, f x), \mathrm{d}(y, f y), \frac{1}{4 s}(\mathrm{~d}(x, f y)+\mathrm{d}(y, f x))\right\}, \quad \text { for all } x, y \in X
$$

Theorem 1. Let $f: X \longrightarrow X$ be a self-map on a $b$-metriclike space $\left(X_{*}, d, s\right)$ with parameter $s \geq 1$. Suppose that there is $\kappa \in K_{\psi-s}$ such that

$$
\kappa(\mathrm{d}(f x, f y), A(x, y)) \geq 0,
$$

for all $x, y \in X$, where $A(x, y)$, is defined as in (8), then the self-map $f$ has a unique fixed point in $X$.
Proof. Let $x_{0} \in X$ be an arbitrary element. Define a sequence $\left\{x_{q}\right\}$ in $X$ such that $\forall q \in N \cup\{0\}, x_{q+1}=f\left(x_{q}\right)$.

If $\mathrm{d}\left(x_{q}, x_{q+1}\right)=0$ for some $q \in N \cup\{0\}$, that is, $x_{q+1}=x_{q}$ and $x_{q}=x_{q+1}=f\left(x_{q}\right)$; therefore, $x_{q}$ is a fixed point of $f$. Thus, suppose that $\mathrm{d}\left(x_{q}, x_{q+1}\right)>0$ for all $q \in N \cup\{0\}$. Considering the set $A(x, y)$, we have

$$
A\left(x_{q-1}, x_{q}\right)=\max \left\{\begin{array}{c}
\mathrm{d}\left(x_{q-1}, x_{q}\right), \mathrm{d}\left(x_{q-1}, f x_{q-1}\right), \mathrm{d}\left(x_{q}, f x_{q}\right) \\
\frac{1}{4 s}\left(\mathrm{~d}\left(x_{q-1}, f x_{q}\right)+\mathrm{d}\left(x_{q}, f x_{q-1}\right)\right) .
\end{array}\right\}=\max \left\{\begin{array}{c}
\mathrm{d}\left(x_{q-1}, x_{q}\right), \mathrm{d}\left(x_{q-1}, x_{q}\right), \mathrm{d}\left(x_{q}, x_{q+1}\right), \\
\frac{1}{4 s}\left(\mathrm{~d}\left(x_{q-1}, x_{q+1}\right)+\mathrm{d}\left(x_{q}, x_{q}\right)\right) .
\end{array}\right\}
$$

Since

we obtain using (10),

$$
\begin{aligned}
& \begin{array}{l}
\frac{1}{4 s}(\mathrm{~d}(x) \\
\operatorname{ng}(10),
\end{array} \\
& A\left(x_{q-1}, x_{q}\right)=\max \left\{\mathrm{d}\left(x_{q-1}, x_{q}\right), \mathrm{d}\left(x_{q}, x_{q+1}\right)\right\} .
\end{aligned}
$$

By the supposition $\mathrm{d}\left(x_{q}, x_{q+1}\right)>0$ and (12), we get $A\left(x_{q-1}, x_{q}\right)>0$. Assume that $A\left(x_{q-1}, x_{q}\right)=\mathrm{d}\left(x_{q}, x_{q+1}\right)$.

Then, applying condition (9) and property $\kappa_{1}$, we have for all $q \in N$

$$
\begin{aligned}
0 \leq & \kappa\left(\mathrm{d}\left(x_{q}, x_{q+1}\right), A\left(x_{q-1}, x_{q}\right)\right) \\
= & \kappa\left(\mathrm{d}\left(f x_{q-1}, f x_{q}\right), A\left(x_{q-1}, x_{q}\right)\right) \\
= & \kappa\left(\mathrm{d}\left(x_{q}, x_{q+1}\right), \mathrm{d}\left(x_{q}, x_{q+1}\right)\right)<\psi\left(\mathrm{d}\left(x_{q}, x_{q+1}\right)\right) \\
& -\psi\left(s^{\lambda} \mathrm{d}\left(x_{q}, x_{q+1}\right)\right) .
\end{aligned}
$$

That is, a contradiction. Therefore,

$$
A\left(x_{q-1}, x_{q}\right)=\mathrm{d}\left(x_{q-1}, x_{q}\right) \text {. }
$$

From (9) and using (14), we obtain
In view of property of $\left(\psi_{1}\right)$, the above inequality gives $\mathrm{d}\left(x_{q}, x_{q+1}\right)<\mathrm{d}\left(x_{q-1}, x_{q}\right)$ for all $q \in N$. Hence, $\left\{\mathrm{d}\left(x_{q}, x_{q+1}\right)\right\}$ is a decreasing sequence of nonnegative reals, so there is $l \geq 0$ so that $\mathrm{d}\left(x_{q}, x_{q+1}\right) \longrightarrow l$. Also, by (14),

$$
\lim _{q \longrightarrow+\infty} \mathrm{d}\left(x_{q}, x_{q+1}\right)=\lim _{q \longrightarrow+\infty} A\left(x_{q-1}, x_{q}\right)=l .
$$

Suppose that $l>0$, then $\quad \lim _{q \longrightarrow+\infty} \mathrm{d}\left(x_{q}\right.$, $\left.x_{q+1}\right)=\lim _{q \longrightarrow+\infty} A\left(x_{q-1}, x_{q}\right)=l>0$. By property $\left(\kappa_{2}\right)$, we have 


$$
0 \leq \limsup _{q \longrightarrow+\infty} \kappa\left(\mathrm{d}\left(x_{q}, x_{q+1}\right), A\left(x_{q-1}, x_{q}\right)\right)<0,
$$

which is a contradiction. Therefore, $l=0$. Hence,

$$
\lim _{q \longrightarrow+\infty} \mathrm{d}\left(x_{q}, x_{q+1}\right)=\lim _{q \longrightarrow+\infty} A\left(x_{q-1}, x_{q}\right)=0 .
$$

$$
\begin{aligned}
\mathrm{d}\left(x_{p_{k}}, x_{q_{k}}\right) \geq \varepsilon, \mathrm{d}\left(x_{p_{k}}, x_{q_{k}-1}\right)<\varepsilon, \frac{\varepsilon}{s^{2}} \leq \limsup _{k \longrightarrow \infty} \mathrm{d}\left(x_{p_{k}-1}, x_{q_{k}-1}\right) \leq \varepsilon s, \\
\frac{\varepsilon}{s} \leq \limsup _{k \longrightarrow \infty} \mathrm{d}\left(x_{q_{k}-1}, x_{p_{k}}\right) \leq \varepsilon \\
\frac{\varepsilon}{s} \leq \limsup _{k \longrightarrow \infty} \mathrm{d}\left(x_{p_{k}-1}, x_{q_{k}}\right) \leq \varepsilon s^{2} .
\end{aligned}
$$

From the definition of $A(x, y)$, we have

$$
\begin{aligned}
A\left(x_{p_{k}-1}, x_{q_{k}-1}\right) & =\max \left\{\mathrm{d}\left(x_{p_{k}-1}, x_{q_{k}-1}\right), \mathrm{d}\left(x_{p_{k}-1}, f x_{p_{k}-1}\right), \mathrm{d}\left(x_{q_{k}-1}, f x_{q_{k}-1}\right), \frac{1}{4 s}\left(\mathrm{~d}\left(x_{p_{k}-1}, f x_{q_{k}-1}\right)+\mathrm{d}\left(x_{q_{k}-1}, f x_{p_{k}-1}\right)\right)\right\} \\
& =\max \left\{\mathrm{d}\left(x_{p_{k}-1}, x_{q_{k}-1}\right), \mathrm{d}\left(x_{p_{k}-1}, x_{p_{k}}\right), \mathrm{d}\left(x_{q_{k}-1}, x_{q_{k}}\right), \frac{1}{4 s}\left(\mathrm{~d}\left(x_{p_{k}-1}, x_{q_{k}}\right)+\mathrm{d}\left(x_{q_{k}-1}, x_{p_{k}}\right)\right)\right\} .
\end{aligned}
$$

By the upper limit $k \longrightarrow+\infty$ in (20) and keeping in mind (18-19), we obtain

$$
\begin{aligned}
\limsup _{k \longrightarrow+\infty} A\left(x_{p_{k}-1}, x_{q_{k}-1}\right) & =\limsup _{k \longrightarrow \infty} \max \left\{\mathrm{d}\left(x_{p_{k}-1}, x_{q_{k}-1}\right), \mathrm{d}\left(x_{p_{k}-1}, x_{p_{k}}\right), \mathrm{d}\left(x_{q_{k}-1}, x_{q_{k}}\right), \frac{1}{4 s}\left(\mathrm{~d}\left(x_{p_{k}-1}, x_{q_{k}}\right)+\mathrm{d}\left(x_{q_{k}-1}, x_{p_{k}}\right)\right)\right\} \\
& \leq \max \left\{\varepsilon s, 0,0, \frac{1}{4 s}\left(\varepsilon s^{2}+\varepsilon\right)\right\} \leq \varepsilon s .
\end{aligned}
$$

Also, from condition $\kappa_{1}$, we have

$$
0 \leq \kappa\left(\mathrm{d}\left(x_{p_{k}}, x_{q_{k}}\right), A\left(x_{p_{k}-1}, x_{q_{k}-1}\right)\right)=\kappa\left(\mathrm{d}\left(x_{p_{k}}, x_{q_{k}}\right), A\left(x_{p_{k}-1}, x_{q_{k}-1}\right)\right)<\psi\left(A\left(x_{p_{k}-1}, x_{q_{k}-1}\right)\right)-\psi\left(s^{\lambda} \mathrm{d}\left(x_{p_{k}}, x_{q_{k}}\right)\right),
$$

which by property of $\left(\psi_{1}\right)$ implies

$$
s^{\lambda} \mathrm{d}\left(x_{p_{k}}, x_{q_{k}}\right)<A\left(x_{p_{k}-1}, x_{q_{k}-1}\right) .
$$

By taking upper limit on both sides of (23) in view of (19) and (21), it follows that

$$
\varepsilon s^{\lambda}<\varepsilon s
$$

which contradicts $\varepsilon>0$. Thus, $\lim _{q, p \longrightarrow+\infty} \mathrm{d}\left(x_{q}, x_{p}\right)=0$ and the sequence $\left\{x_{q}\right\}$ is Cauchy in $\left(X_{*}, d, s\right)$. So, there is $\omega \in X$, such that

$$
\lim _{q \longrightarrow+\infty} \mathrm{d}\left(x_{q}, \omega\right)=\mathrm{d}(\omega, \omega)=\lim _{q, p \longrightarrow+\infty} \mathrm{d}\left(x_{q}, x_{p}\right)=0 .
$$

For elements $\omega$ and $x_{q}$, we consider 


$$
\begin{aligned}
A\left(x_{q}, \omega\right) & =\max \left\{\mathrm{d}\left(x_{q}, \omega\right), \mathrm{d}\left(x_{q}, f x_{q}\right), \mathrm{d}(\omega, f \omega), \frac{1}{4 s}\left(\mathrm{~d}\left(x_{q}, f \omega\right)+\mathrm{d}\left(\omega, f x_{q}\right)\right)\right\} \\
& =\max \left\{\mathrm{d}\left(x_{q}, \omega\right), \mathrm{d}\left(x_{q}, x_{q+1}\right), \mathrm{d}(\omega, f \omega), \frac{1}{4 s}\left(\mathrm{~d}\left(x_{q}, f \omega\right)+\mathrm{d}\left(\omega, x_{q+1}\right)\right)\right\} .
\end{aligned}
$$

By Lemma 1 together with (18) and (25), it follows by passing in the upper limit of (26):

$$
\lim _{q \longrightarrow+\infty} \sup A\left(x_{q}, \omega\right) \leq \max \left\{0,0, \mathrm{~d}(\omega, f \omega), \frac{s \mathrm{~d}(\omega, f \omega)}{4 s}\right\}=\mathrm{d}(\omega, f \omega) .
$$

Now, using the $\kappa_{1}$ condition, we have

$$
\begin{array}{r}
0 \leq \kappa\left(\mathrm{d}\left(x_{q+1}, f \omega\right), A\left(x_{q}, \omega\right)=\kappa\left(\mathrm{d}\left(f x_{q}, f \omega\right), A\left(x_{q}, \omega\right)\right)\right)<\psi\left(A\left(x_{q}, \omega\right)\right)-\psi\left(s^{\lambda} \mathrm{d}\left(x_{q+1}, f \omega\right)\right), \\
s^{\lambda-1} \mathrm{~d}(\omega, f \omega)=s^{\lambda} \cdot \frac{1}{s} \mathrm{~d}(\omega, f \omega)<\mathrm{d}(\omega, f \omega) .
\end{array}
$$

which implies

$$
s^{\lambda} \mathrm{d}\left(x_{q+1}, f \omega\right)<A\left(x_{q}, \omega\right)
$$

Taking the limit superior in (29) and by Lemma 1 and inequality (27), we obtain

By (30), it follows that $\mathrm{d}(\omega, f \omega)=0$, and so $f \omega=\omega$.

Suppose $\omega, y \in X$ are two different fixed points of $f$. By (14), we have $\mathrm{d}(\omega, \omega)=0$ (and also $\mathrm{d}(y, y)=0)$. Since $\omega \neq y(\mathrm{~d}(\omega, y)>0)$, one writes

$$
A(\omega, y)=\max \left\{\mathrm{d}(\omega, y), \mathrm{d}(\omega, \omega), \mathrm{d}(y, y), \frac{1}{4 s}(\mathrm{~d}(\omega, y)+\mathrm{d}(y, \omega))\right\}=\max \left\{\mathrm{d}(\omega, y), 0,0, \frac{1}{2 s} \mathrm{~d}(\omega, y)\right\}=\mathrm{d}(\omega, y)>0
$$

From condition (9) and property $\kappa_{1}$, we have

$$
0 \leq \kappa(\mathrm{d}(f \omega, f y), A(\omega, y))=\kappa(\mathrm{d}(\omega, y), \mathrm{d}(\omega, y))<\psi(\mathrm{d}(\omega, y))-\psi\left(s^{\lambda} \mathrm{d}(\omega, y)\right),
$$

which is a contradiction. Therefore, $\mathrm{d}(\omega, y)=0$ and $\omega=y$. Thus, there is a unique fixed point of $f$.

Example 2. Let $X=[0,1]$ with the $b$-metric-like $d(x, y)=(x+y)^{2}$. Define $f: X \longrightarrow X$ as $f x=$ $\left\{\begin{array}{ll}(1 / 6) x & \text { if } x \neq 1 \\ (1 / 8) & \text { if } x=1\end{array}\right.$.
Also, we take the functions $\phi(x)=x ; \psi(x)=2 x$ and $\kappa(t, v)=\phi(v)-\psi\left(s^{\lambda} t\right)$, (where $\left.\lambda=2\right)$ for all $t, v \in(0,+\infty)$, where $\psi ; \phi:[0,+\infty) \longrightarrow[0,+\infty)$ are continuous and $\psi$ is increasing such that $\phi(v)<\psi(v)$ for all $v>0$.

The pair $(X, d)$ is a $b$ - metric-like space with coefficient $s=2$. We claim that the mapping $f$ satisfies the contraction type condition (8):

Case1. For $x \neq y \neq 1$, we have 


$$
\begin{aligned}
A(x, y) & =\max \left\{\mathrm{d}(x, y), \mathrm{d}(x, f x) \mathrm{d}(y, f y) \frac{1}{4 s}(\mathrm{~d}(x, f y)+\mathrm{d}(y, f x))\right\} \\
& =\max \left\{(x+y)^{2},\left(x+\frac{x}{6}\right)^{2},\left(y+\frac{y}{6}\right)^{2}, \frac{1}{4 s}\left(\left(x+\frac{y}{6}\right)^{2}+\left(y+\frac{x}{6}\right)^{2}\right)\right\} .
\end{aligned}
$$

And $\mathrm{d}(f x, f y)=\mathrm{d}(x / 6, y / 6)=(x / 6, y / 6)^{2}=1 / 36(x+$ $y)^{2}=(1 / 36) \mathrm{d}(x, y)$. Then,

$$
\begin{aligned}
\kappa(\mathrm{d}(f x, f y), A(x, y)) & =\phi(A(x, y))-\psi\left(s^{2} d(f x, f y)\right)=A(x, y)-2 s^{2} \mathrm{~d}(f x, f y)=A(x, y)-8 \mathrm{~d}(f x, f y) \\
& =A(x, y)-8 \frac{1}{36} \mathrm{~d}(x, y)=A(x, y)-\frac{2}{9} \mathrm{~d}(x, y) \geq 0 .
\end{aligned}
$$

Case 2. For $x=y=1$, we note

$$
\begin{aligned}
A(1,1) & =\max \left\{\mathrm{d}(1,1), \mathrm{d}(1, f 1), \mathrm{d}(1, f 1), \frac{1}{4 s}(\mathrm{~d}(1, f 1)+\mathrm{d}(1, f 1))\right\}=\max \{\mathrm{d}(1,1), \mathrm{d}(1, f 1)\} \\
& =\max \left\{(1+1)^{2},\left(1+\frac{1}{8}\right)^{2}\right\}=4=\mathrm{d}(1,1) .
\end{aligned}
$$

And $\mathrm{d}(f 1, f 1)=\mathrm{d}(1 / 8,1 / 8)=(1 / 8,+1 / 8)^{2}=4 / 64=$ $(1 / 64) \mathrm{d}(1,1)<A(1,1)$. Then,

$$
\begin{aligned}
\kappa(\mathrm{d}(f 1, f 1), A(1,1)) & =\phi(A(1,1))-\psi\left(s^{2} \mathrm{~d}(f 1, f 1)\right)=A(1,1)-2 s^{2} \mathrm{~d}(f 1, f 1)=A(1,1)-8 \mathrm{~d}(f 1, f 1)=A(1,1)-8 \frac{1}{64} \mathrm{~d}(1,1) \\
& =A(1,1)-\frac{2}{9} \mathrm{~d}(1,1) \geq 0 .
\end{aligned}
$$

Case 3. $x<y=1$ we note

$$
\begin{aligned}
\mathrm{d}(f x, f 1) & =\mathrm{d}\left(\frac{x}{6}, \frac{1}{8}\right)=\left(\frac{x}{6}+\frac{1}{8}\right)^{2}<\frac{1}{36}(x+1)^{2}=\frac{1}{36} \mathrm{~d}(x, 1) \\
& \Longrightarrow \mathrm{d}(f x, f 1)<\frac{1}{36} \mathrm{~d}(x, 1) \\
& \Longrightarrow 8 \mathrm{~d}(f x, f 1)<8 \frac{1}{36} \mathrm{~d}(x, 1) \\
& \Longrightarrow 2 s^{2} \mathrm{~d}(f x, f 1)<\frac{2}{9} \mathrm{~d}(x, 1)<A(x, 1)
\end{aligned}
$$

Then,

$$
\kappa(\mathrm{d}(f x, f 1), A(x, 1))=\phi(A(x, 1))-\psi\left(s^{2} \mathrm{~d}(f x, f 1)\right)=A(x, 1)-2 s^{2} \mathrm{~d}(f x, f 1) \geq 0 .
$$


Here, 0 is the unique fixed point of $f$.

Some applications of Theorem 1 are the following corollaries.

Corollary 1. Let $f: X \longrightarrow X$ be a mapping on a $b$-metriclike space $\left(X_{*}, d, s\right)$. Suppose that there are $\psi \in \Psi$ and $\lambda \geq 1$ such that

$$
\psi\left(s^{\lambda} d(f x, f y)\right) \leq \frac{\psi(A(x, y))}{1+\psi(A(x, y))},
$$

for all $x, y \in X$, where $A(x, y)$ is defined as in (8). Then, the self-map $f$ has a unique fixed point in $X$.

Proof. In Theorem 1, take into account the function $\kappa(t, v)=$ $\psi(v) /(1+\psi(v))-\psi\left(s^{\lambda} t\right)$ for all $t, v \in(0,+\infty)$,

Corollary 2. Let $f: X \longrightarrow X$ be a mapping on a $b$-metriclike space $\left(X_{*}, d, s\right)$. Suppose that there are $\psi \in \Psi$, $\varphi:[0,+\infty) \longrightarrow[0,+\infty)$ a lower semicontinuous function with $\varphi(v)=0$ iff $v=0$, and $\lambda \geq 1$ such that

$$
\psi\left(s^{\lambda} d(f x, f y)\right) \leq \frac{\psi(A(x, y))}{1+\varphi(A(x, y))},
$$

for all $x, y \in X$, where $A(x, y)$ is defined as in (8). Then, the self-mapf admits a unique fixed point in $X$.

Proof. In Theorem 1, take into account the function $\kappa(t, v)=$ $\psi(v) /(1+\varphi(v))-\psi\left(s^{\lambda} t\right)$ for all $t, v \in(0,+\infty)$.

Corollary 3. Let $f: X \longrightarrow X$ be a mapping on a b-metriclike space $\left(X_{*}, d, s\right)$. Suppose that there are $\psi \in \Psi, \alpha \in(0,1)$ and $\lambda \geq 1$ such that

$$
\psi\left(s^{\lambda} \mathrm{d}(f x, f y)\right) \leq \alpha \psi(A(x, y))
$$

for all $x, y \in X$, where $A(x, y)$ is defined as in (8). Then, the self-map $f$ has a unique fixed point in $X$.

Proof. In Theorem 1, take into account the function $\kappa(t, v)=\alpha \psi(v)-\psi\left(s^{\lambda} t\right)$ for all $t, v \in(0,+\infty)$, and $\alpha \in(0,1)$.

Corollary 4. Let $f: X \longrightarrow X$ be a mapping on a $b$-metriclike space $\left(X_{*}, d, s\right)$. Suppose that there are $\psi \in \Psi, \lambda \geq 1$, and $\phi: R^{+} \longrightarrow R^{+}$continuous with $\phi(v)<\psi(v)$ for $v>0$, such that

$$
\psi\left(s^{\lambda} \mathrm{d}(f x, f y)\right) \leq \phi(A(x, y)),
$$

for all $x, y \in X$, where $A(x, y)$ is defined as in (8). Then, the self-mapf has a unique fixed point in $X$.

Proof. In Theorem 1, take into account the function $\kappa(t, v)=\phi(v)-\psi\left(s^{\lambda} t\right)$ for all $t, v \in(0,+\infty)$.

Corollary 5. Let $f: X \longrightarrow X$ be a mapping on a b-metriclike space $\left(X_{*}, d, s\right)$. Suppose that there are $\psi \in \Psi, \lambda \geq 1$, $F: R^{+} \times R^{+} \longrightarrow R$ a C-class function and $\varphi: R^{+} \longrightarrow R^{+} a$ continuous function, such that

$$
\psi\left(s^{\lambda} \mathrm{d}(f x, f y)\right) \leq F(\psi(A(x, y), \varphi(A(x, y)))),
$$

for all $x, y \in X$, where $A(x, y)$ is defined as in (8). Then, the self-map $f$ has a unique fixed point in $X$.

Proof. In Theorem 1, take into account the function $\kappa(t, v)=F(\psi(v), \varphi(v))-\psi\left(s^{\lambda} t\right)$ for all $t, v \in(0,+\infty)$, where $F: R^{+} \times R^{+} \longrightarrow R$ is a C-class function.

Remark 2. Corollary 5 is much wider because condition (43) includes many other contractive conditions.

Corollary 6. Let $f: X \longrightarrow X$ be a mapping on a $b$-metriclike space $\left(X_{*}, d, s\right)$. Suppose that there exist a function $\varphi:[0,+\infty) \longrightarrow[0,+\infty)$ with $\liminf _{t \longrightarrow v} \varphi(t)>0$ for all $v>0$, and some constant $\lambda \geq 1$ such that

$$
s^{\lambda} \mathrm{d}(f x, f y) \leq A(x, y)-\varphi(A(x, y)),
$$

for all $x, y \in X$, where $A(x, y)$ is defined as in (8). Then, the self-map $f$ has a unique fixed point in $X$.

Proof. In Theorem 1, take into account the function $\kappa(t, v)=\psi(v)-\varphi(v)-\psi\left(s^{\lambda} t\right)$ for all $t, v \in(0,+\infty)$, and take $\psi(v)=v$ (it corresponds to Theorem 3.16 in [23]).

In the following result, we include two mappings $f$ and $g$ in the set

$$
\begin{aligned}
& E(x, y)=\max \\
& \left\{\mathrm{d}(x, y), \mathrm{d}(y, g y), \frac{\mathrm{d}(x, f x) \mathrm{d}(y, g y)}{1+\mathrm{d}(x, y)}, \frac{\mathrm{d}(x, f x) \mathrm{d}(y, g y)}{1+\mathrm{d}(f x, g y)}\right\} .
\end{aligned}
$$


Theorem 2. Let $\left(X_{*}, d, s\right)$ be a b-metric-like space and $f, g: X \longrightarrow X$ be two given mappings. Suppose that there exists $\kappa \in K_{\psi-s}$ such that

$$
\kappa(\mathrm{d}(f x, g y), E(x, y)) \geq 0,
$$

Proof. Let $x_{0} \in X$ be an arbitrary element. Define a sequence $\left\{x_{q}\right\}$ in $X$ such that $\forall q \in N \cup\{0\} x_{2 q+1}=f x_{2 q}$ and $x_{2 q+2}=g x_{2 q+1}$.

Let for some $q \in \mathbb{N} \cup\{0\} x_{2 q+1}=x_{2 q}$. Since

for all $x, y \in X$, where $E(x, y)$ is denoted by (45); then, the mappings $f$ and $g$ have a unique common fixed point in $X$.

$$
\begin{aligned}
E\left(x_{2 q}, x_{2 q+1}\right) & =\max \left\{\mathrm{d}\left(x_{2 q}, x_{2 q+1}\right), \mathrm{d}\left(x_{2 q+1}, g x_{2 q+1}\right), \frac{\mathrm{d}\left(x_{2 q}, f x_{2 q}\right) \mathrm{d}\left(x_{2 q+1}, g x_{2 q+1}\right)}{1+\mathrm{d}\left(x_{2 q}, x_{2 q+1}\right)}, \frac{\mathrm{d}\left(x_{2 q}, f x_{2 q}\right) \mathrm{d}\left(x_{2 q+1}, g x_{2 q+1}\right)}{1+\mathrm{d}\left(f x_{2 q}, g x_{2 q+1}\right)}\right\} \\
& =\max \left\{\mathrm{d}\left(x_{2 q}, x_{2 q+1}\right), \mathrm{d}\left(x_{2 q+1}, x_{2 q+2}\right), \frac{\mathrm{d}\left(x_{2 q}, x_{2 q+1}\right) \mathrm{d}\left(x_{2 q+1}, x_{2 q+2}\right)}{1+\mathrm{d}\left(x_{2 q}, x_{2 q+1}\right)}, \frac{\mathrm{d}\left(x_{2 q}, x_{2 q+1}\right) \mathrm{d}\left(x_{2 q+1}, x_{2 q+2}\right)}{1+\mathrm{d}\left(x_{2 q+1}, x_{2 q+2}\right)}\right\} \\
& =\max \left\{0, \mathrm{~d}\left(x_{2 q+1}, x_{2 q+2}\right), 0,0\right\} \\
& =\mathrm{d}\left(x_{2 q+1}, x_{2 q+2}\right) .
\end{aligned}
$$

Then, by $(46)$ and $\left(\kappa_{1}\right)$, we have

$$
0 \leq \kappa\left(\mathrm{d}\left(f x_{2 q}, g x_{2 q+1}\right), E\left(x_{2 q}, x_{2 q+1}\right)\right)=\kappa\left(\mathrm{d}\left(x_{2 q+1}, x_{2 q+2}\right), \mathrm{d}\left(x_{2 q+1}, x_{2 q+2}\right)\right)<\psi\left(\mathrm{d}\left(x_{2 q+1}, x_{2 q+2}\right)\right)-\psi\left(s^{\lambda} \mathrm{d}\left(x_{2 q+1}, x_{2 q+2}\right)\right) .
$$

By property $\left(\psi_{1}\right)$, we get $d\left(x_{2 q+1}, x_{2 q+2}\right)=0$, that is, $x_{2 q+1}=x_{2 q+2}$. We deduce that $x_{2 q}=x_{2 q+1}=f x_{2 q}$ and $g x_{2 q}=g f x_{2 q}=g x_{2 q+1}=x_{2 q+2}=x_{2 q}$. Hence, $x_{2 q}$ is a common fixed point of $f$ and $g$.
Assume the general case that $\mathrm{d}\left(x_{2 q}, x_{2 q+1}\right)>0$ for all $q \in N \cup\{0\}$, then

$$
\begin{aligned}
E\left(x_{2 q}, x_{2 q-1}\right) & =\max \left\{\mathrm{d}\left(x_{2 q}, x_{2 q-1}\right), \mathrm{d}\left(x_{2 q-1}, g x_{2 q-1}\right), \frac{\mathrm{d}\left(x_{2 q}, f x_{2 q}\right) \mathrm{d}\left(x_{2 q-1}, g x_{2 q-1}\right)}{1+\mathrm{d}\left(x_{2 q}, x_{2 q-1}\right)}, \frac{\mathrm{d}\left(x_{2 q}, f x_{2 q}\right) \mathrm{d}\left(x_{2 q-1}, g x_{2 q-1}\right)}{1+\mathrm{d}\left(f x_{2 q}, g x_{2 q-1}\right)}\right\} \\
& =\max \left\{\mathrm{d}\left(x_{2 q}, x_{2 q-1}\right), \mathrm{d}\left(x_{2 q-1}, x_{2 q}\right), \frac{\mathrm{d}\left(x_{2 q}, x_{2 q+1}\right) \mathrm{d}\left(x_{2 q-1}, x_{2 q}\right)}{1+\mathrm{d}\left(x_{2 q}, x_{2 q-1}\right)}, \frac{\mathrm{d}\left(x_{2 q}, x_{2 q+1}\right) \mathrm{d}\left(x_{2 q-1}, x_{2 q}\right)}{1+\mathrm{d}\left(x_{2 q+1}, x_{2 q}\right)}\right\} \\
& =\max \left\{\mathrm{d}\left(x_{2 q}, x_{2 q-1}\right), \mathrm{d}\left(x_{2 q}, x_{2 q+1}\right)\right\} . \\
\text { If } \mathrm{d}\left(x_{2 q}\right. & E\left(x_{2 q-1}, x_{2 q}\right)=\mathrm{d}\left(x_{2 q}, x_{2 q+1}\right)>0 .
\end{aligned}
$$

If $\mathrm{d}\left(x_{2 q-1}, x_{2 q}\right) \leq \mathrm{d}\left(x_{2 q}, x_{2 q+1}\right)$ for some $q \in \mathrm{N}$, then (49) implies
From (50), applying $\left(\psi_{1}\right),(46)$, and $\left(\kappa_{1}\right)$, we have

$$
\begin{aligned}
0 \leq \kappa\left(\mathrm{d}\left(x_{2 q+1}, x_{2 q}\right), E\left(x_{2 q}, x_{2 q-1}\right)\right) & =\kappa\left(\mathrm{d}\left(f x_{2 q}, g x_{2 q-1}\right), E\left(x_{2 q}, x_{2 q-1}\right)\right)<\psi\left(E\left(x_{2 q}, x_{2 q-1}\right)\right)-\psi\left(s^{\lambda} \mathrm{d}\left(f x_{2 q}, g x_{2 q-1}\right)\right) \\
& =\psi\left(\mathrm{d}\left(x_{2 q+1}, x_{2 q}\right)\right)-\psi\left(s^{\lambda} \mathrm{d}\left(x_{2 q+1}, x_{2 q}\right)\right) \leq 0 .
\end{aligned}
$$


That is a contradiction. So, we have $\left\{\mathrm{d}\left(x_{2 q+1}, x_{2 q}\right)\right\}$ is a decreasing sequence of nonnegative reals, $\mathrm{d}\left(x_{2 q}, x_{2 q+1}\right)<\mathrm{d}\left(x_{2 q-1}, x_{2 q}\right)$ for all $q \in N$ Hence, so there is $l \geq 0$ so that

$$
\lim _{q \longrightarrow+\infty} \mathrm{d}\left(x_{q}, x_{q+1}\right)=l \text { and also } \lim _{q \longrightarrow+\infty} \mathrm{d}\left(x_{q}, x_{q+1}\right)=\lim _{q \longrightarrow+\infty} E\left(x_{q-1}, x_{q}\right)=l \text {. }
$$

Assume that $l>0$; then, by applying $\kappa_{2}$, we have

$$
\underset{q \longrightarrow+\infty}{\limsup } \kappa\left(\mathrm{d}\left(x_{2 q+1}, x_{2 q}\right), E\left(x_{2 q}, x_{2 q-1}\right)\right) \leq 0,
$$

a contradiction. Therefore,

$$
\lim _{q \longrightarrow+\infty} \mathrm{d}\left(x_{q}, x_{q+1}\right)=\lim _{q \longrightarrow+\infty} E\left(x_{q-1}, x_{q}\right)=l>0 .
$$

Now, we prove that $\lim _{q, p \longrightarrow+\infty} \mathrm{d}\left(x_{q}, x_{p}\right)=0$. It is enough to prove that $\lim _{q, p \longrightarrow+\infty} \mathrm{d}\left(x_{2 q}, x_{2 p}\right)=0$ On the contrary, assume that $\lim _{q, p \longrightarrow+\infty} \mathrm{d}\left(x_{2 q}, x_{2 p}\right) \neq 0$. Then, from Lemma 2, there are $\varepsilon>0$ and two subsequences $\left\{p_{k}\right\}$ and $\left\{q_{k}\right\}$ of positive integers, with $q_{k}>p_{k}>k$, such that

$\varepsilon \leq \limsup _{k \longrightarrow \infty} \mathrm{d}\left(x_{2 q_{k}}, x_{2 p_{k}}\right) \leq \varepsilon s, \frac{\varepsilon}{s} \leq \limsup _{k \longrightarrow \infty} \mathrm{d}\left(x_{2 p_{k}}, x_{2 q_{k}-1}\right) \leq \varepsilon s, \frac{\varepsilon}{s^{2}} \leq \limsup _{k \longrightarrow \infty} \mathrm{d}\left(x_{2 q_{k}-1}, x_{2 p_{k}+1}\right) \leq \varepsilon s^{2}, \frac{\varepsilon}{s} \leq \limsup _{k \longrightarrow \infty} \mathrm{d}\left(x_{2 p_{k}+1}, x_{2 q_{k}}\right) \leq \varepsilon s^{2}$.

From (45), we note

$$
\begin{aligned}
E\left(x_{2 p_{k}}, x_{2 q_{k}-1}\right) & =\max \left\{\mathrm{d}\left(x_{2 p_{k}}, x_{2 q_{k}-1}\right), \mathrm{d}\left(x_{2 q_{k}-1}, g x_{2 q_{k}-1}\right), \frac{\mathrm{d}\left(x_{2 p_{k}}, f x_{2 p_{k}}\right) \mathrm{d}\left(x_{2 q_{k}-1}, g x_{2 q_{k}-1}\right)}{1+\mathrm{d}\left(x_{2 p_{k}}, x_{2 q_{k}-1}\right)}, \frac{\mathrm{d}\left(x_{2 p_{k}}, f x_{2 p_{k}}\right) \mathrm{d}\left(x_{2 q_{k}-1}, g x_{2 p_{k}-1}\right)}{1+\mathrm{d}\left(f x_{2 p_{k}}, g x_{2 q_{k}-1}\right)}\right\} \\
& =\max \left\{\mathrm{d}\left(x_{2 p_{k}}, x_{2 q_{k}-1}\right), \mathrm{d}\left(x_{2 q_{k}-1}, x_{2 q_{k}}\right), \frac{\mathrm{d}\left(x_{2 p_{k}}, x_{2 p_{k}+1}\right) \mathrm{d}\left(x_{2 q_{k}-1}, x_{2 q_{k}}\right)}{1+\mathrm{d}\left(x_{2 p_{k}}, x_{2 q_{k}-1}\right)}, \frac{\mathrm{d}\left(x_{2 p_{k}}, x_{2 p_{k}+1}\right) \mathrm{d}\left(x_{2 q_{k}-1}, x_{2 q_{k}}\right)}{1+\mathrm{d}\left(x_{2 p_{k}+1}, x_{2 q_{k}}\right)}\right\} .
\end{aligned}
$$

Hence, by (54)-(56), and Lemma 2, we have

$$
\begin{aligned}
\limsup _{k \longrightarrow+\infty} E\left(x_{2 p_{k}}, x_{2 q_{k}-1}\right) & =\limsup _{k \longrightarrow+\infty} \max \left\{\mathrm{d}\left(x_{2 p_{k}}, x_{2 q_{k}-1}\right), \mathrm{d}\left(x_{2 q_{k}-1}, x_{2 q_{k}}\right), \frac{\mathrm{d}\left(x_{2 p_{k}}, x_{2 p_{k}+1}\right) \mathrm{d}\left(x_{2 q_{k}-1}, x_{2 q_{k}}\right)}{1+\mathrm{d}\left(x_{2 p_{k}}, x_{2 q_{k}-1}\right)}, \frac{\mathrm{d}\left(x_{2 p_{k}}, x_{2 p_{k}+1}\right) \mathrm{d}\left(x_{2 q_{k}-1}, x_{2 q_{k}}\right)}{1+\mathrm{d}\left(x_{2 p_{k}+1}, x_{2 q_{k}}\right)}\right\} \\
& \leq \max \{\varepsilon s, 0,0,0\}=\varepsilon s .
\end{aligned}
$$

By (46) and using properties $\left(\psi_{1}\right),\left(\kappa_{1}\right)$, we have

$$
0 \leq \kappa\left(\mathrm{d}\left(f x_{2 p_{k}}, g x_{2 q_{k}-1}\right), E\left(x_{2 p_{k}}, x_{2 q_{k}-1}\right)\right)=\kappa\left(\mathrm{d}\left(x_{2 p_{k}+1}, x_{2 q_{k}}\right), E\left(x_{2 p_{k}}, x_{2 q_{k}-1}\right)\right)<\psi\left(E\left(x_{2 p_{k}}, x_{2 q_{k}-1}\right)\right)-\psi\left(s^{\lambda} \mathrm{d}\left(x_{2 p_{k}+1}, x_{2 q_{k}}\right)\right)
$$


which leads to

$$
s^{\lambda} \mathrm{d}\left(x_{2 p_{k}+1}, x_{2 q_{k}}\right)<E\left(x_{2 p_{k}}, x_{2 q_{k}-1}\right) .
$$

Hence, by (55), (57), and (58) and taking the upper limit, we obtain

$$
\varepsilon s^{\lambda-1}<\varepsilon S,
$$

which implies that $\varepsilon=0$, a contradiction with $\varepsilon>0$. It remains that $\lim _{q, p \longrightarrow+\infty} \mathrm{d}\left(x_{q}, x_{p}\right)=0$; therefore, $\left\{x_{q}\right\}$ is a Cauchy sequence in $X$. Since $\left(X_{*}, d, s\right)$ is a complete $b$-metric-like space, there is $\omega \in X$ such that $\left\{x_{q}\right\}$ is convergent to $\omega$, that is,

$$
\lim _{q \longrightarrow+\infty} \mathrm{d}\left(x_{q}, \omega\right)=\lim _{q, p \longrightarrow+\infty} \mathrm{d}\left(x_{q}, x_{p}\right)=\mathrm{d}(\omega, \omega)=0 .
$$

so

Also, the subsequences $\left\{f x_{2 q}\right\},\left\{g x_{2 q+1}\right\}$ are convergent,

$$
\begin{aligned}
& \lim _{q \longrightarrow+\infty} \mathrm{d}\left(x_{2 q+1}, \omega\right)=\lim _{q \longrightarrow+\infty} \mathrm{d}\left(f x_{2 q}, \omega\right)=\mathrm{d}(\omega, \omega)=0, \\
& \lim _{q \longrightarrow+\infty} \mathrm{d}\left(x_{2 q+2}, \omega\right)=\lim _{q \longrightarrow+\infty} \mathrm{d}\left(g x_{2 q+1}, \omega\right)=\mathrm{d}(\omega, \omega)=0 .
\end{aligned}
$$

Consider

$$
\begin{aligned}
E\left(x_{2 q}, \omega\right) & =\max \left\{\mathrm{d}\left(x_{2 q}, \omega\right), \mathrm{d}(\omega, g \omega), \frac{\mathrm{d}\left(x_{2 q}, f x_{2 q}\right) \mathrm{d}(\omega, g \omega)}{1+\mathrm{d}\left(x_{2 q}, \omega\right)}, \frac{\mathrm{d}\left(x_{2 q}, f x_{2 q}\right) \mathrm{d}(\omega, g \omega)}{1+\mathrm{d}\left(f x_{2 q}, g \omega\right)}\right\} \\
& =\max \left\{\mathrm{d}\left(x_{2 q}, \omega\right), \mathrm{d}(\omega, g \omega), \frac{\mathrm{d}\left(x_{2 q}, x_{2 q+1}\right) \mathrm{d}(\omega, g \omega)}{1+\mathrm{d}\left(x_{2 q}, \omega\right)}, \frac{\mathrm{d}\left(x_{2 q}, x_{2 q+1}\right) \mathrm{d}(\omega, g \omega)}{1+\mathrm{d}\left(x_{2 q+1}, g \omega\right)}\right\} .
\end{aligned}
$$

Taking the limit superior in (63) and applying Lemma 1 and (62), it follows

From condition (46),

$\limsup _{q \longrightarrow+\infty} E\left(x_{2 q}, \omega\right) \leq \max \{0, \mathrm{~d}(\omega, g \omega), 0,0\}=,\mathrm{d}(\omega, g \omega)$.

$$
0 \leq \kappa\left(\mathrm{d}\left(f x_{2 q}, g \omega\right), E\left(x_{2 q}, \omega\right)\right)=\kappa\left(\mathrm{d}\left(x_{2 q+1}, g \omega\right), E\left(x_{2 q}, \omega\right)\right)<\psi\left(E\left(x_{2 q}, \omega\right)\right)-\psi\left(s^{\lambda} \mathrm{d}\left(x_{2 q+1}, g \omega\right)\right)
$$

which implies

$$
s^{\lambda} \mathrm{d}\left(x_{2 q+1}, g \omega\right)<E\left(x_{2 q}, \omega\right) .
$$

Taking the upper limit as $q \longrightarrow+\infty$ and using Lemma 1 and (64), we have $s^{\lambda-1} \mathrm{~d}(\omega, g \omega)<\mathrm{d}(\omega, g \omega)$, that is, $\mathrm{d}(\omega, g \omega)=0$ and $\omega$ is a fixed point of $g$. Similarly, we can get $\mathrm{d}(f \omega, \omega)=0$ and so $\omega$ is a common fixed point for mappings $f$ and $g$. Suppose $\omega, \delta \in X$ are two different common fixed points of $f$ and $g$ such that $\mathrm{d}(\omega, \delta)>0$. Then,

$$
\begin{aligned}
E(\omega, \delta) & =\max \left\{\mathrm{d}(\omega, \delta), \mathrm{d}(\delta, g \delta), \frac{\mathrm{d}(\omega, \omega) \mathrm{d}(\delta, g \delta)}{1+\mathrm{d}(\omega, \delta)}, \frac{\mathrm{d}(\omega, \omega) \mathrm{d}(\delta, g \delta)}{1+\mathrm{d}(f \omega, g \delta)}\right\}=\max \left\{\mathrm{d}(\omega, \delta), \mathrm{d}(\delta, \delta), \frac{\mathrm{d}(\omega, \omega) \mathrm{d}(\delta, \delta)}{1+\mathrm{d}(\omega, \delta)}, \frac{\mathrm{d}(\omega, \omega) \mathrm{d}(\delta, \delta)}{1+\mathrm{d}(\omega, \delta)}\right\} \\
& =\mathrm{d}(\omega, \delta)>0 .
\end{aligned}
$$

From $\left(\psi_{1}\right),\left(\kappa_{1}\right),(67)$, and (46), we have

$$
0 \leq \kappa(\mathrm{d}(f \omega, g \delta), E(\omega, \delta))=\kappa(\mathrm{d}(\omega, \delta), \mathrm{d}(\omega, \delta))<\psi(\mathrm{d}(\omega, \delta))-\psi\left(s^{\lambda} \mathrm{d}(\omega, \delta)\right) \leq 0
$$


which contradicts the supposition $\mathrm{d}(\omega, \delta)>0$. Hence, $\mathrm{d}(\omega, \delta)=0$ and the common fixed point is unique.

Corollary 7. Let $f, g: X \longrightarrow X$ be two self-mappings given in a $b$-metric-like space $\left(X_{*}, d, s\right)$. Suppose that there exist $\psi \in \Psi$ and $\alpha:[0, \infty) \longrightarrow[0,1)$ with $\lim _{t \rightarrow r^{+}} \alpha(t)<1$ for all $r>0$, such that

$$
\psi\left(s^{\lambda} \mathrm{d}(f x, g y)\right) \leq \alpha(E(x, y)) \psi(E(x, y)),
$$

for all $x, y \in X$, where $E(x, y)$ is defined as in (45).

Then, the self-mappings $f$ and $g$ have a unique common fixed point in $X$.

Proof. In Theorem 2, take the $\Psi-s$ simulation function $\kappa(t, v)=\alpha(v) \psi(v)-\psi\left(s^{\lambda} t\right)$ for all $t, v \in(0,+\infty)$.

Remark 3. The above theorem reduces to a one mapping if we put $g=f$. Further corollaries can be stated for $s=1$, either by taking the function $\psi$ as an identity function or by taking different functions $\kappa \in K_{\psi-s}$ as listed in Corollary 1-6.

\section{Conclusion}

In this work, we established common fixed point results for one and two mappings on a $b$-metric-like space which overcomes and unifies classical and previous results developed in papers [19-28]. The considered set of generalized contractive mappings contains the families of many contractions as a proper subset. We remark based on Example 2/ (4) which are functions of $C$-class used by many researchers and taken as a special case of $\Psi-s$ simulation functions.

By using additional set of functions $\Psi, \phi$, coefficient $\lambda$, and parameter $s$, the rich class of $\Psi-s$ simulation functions make it possible to collect, extend, and complement previously existing results related to a variety types of contractions.

In terms of $\Psi-s$ simulating functions, many classical and still recent contractions take a simple form as $\kappa(d(f x, f y), A(x, y)) \geq 0$ not including other additional symbols and long formulas.

This wide approach reflects a wide work and an unifying power for more general theorems made on the theory of fixed points.

\section{Data Availability}

No data were used to support this study.

\section{Conflicts of Interest}

The authors declare that they have no competing interests.

\section{Authors' Contributions}

The authors declare that the study was realized in collaboration with the same responsibility. All authors read and approved the final manuscript. All contributed equally to the writing of this paper.

\section{Acknowledgments}

The second author would like to thank Prince Sultan University for funding this work through research group Nonlinear Analysis Methods in Applied Mathematics (NAMAM) group number RG-DES-2017-01-17.

\section{References}

[1] S. Banach, "Sur les opérations dans les ensembles abstraits et leur application aux équations intégrales," Fundamenta Mathematicae, vol. 3, pp. 133-181, 1922.

[2] F. Khojasteh, S. Shukla, and S. Radenovic, "A new approach to the study of fixed point theory for simulation functions," Filomat, vol. 29, no. 6, pp. 1189-1194, 2015.

[3] H. Argoubi, B. Samet, and C. Vetro, "Nonlinear contractions involving simulation functions in a metric space with a partial order," Journal of Nonlinear Sciences and Applications, vol. 08, no. 06, pp. 1082-1094, 2015.

[4] H. Aydi, H. Lakzian, Z. D. Mitrović, and S. Radenović, "Best proximity points of MT-cyclic contractions with property UC," Numerical Functional Analysis and Optimization, vol. 41, no. 7, pp. 871-882, 2020.

[5] I. A. Bakhtin, "The contraction mapping principle in quasi metric spaces," Functional Analysis, Unianowsk Gos. Ped. Inst.vol. 30, pp. 26-37, 1989.

[6] S. Czerwik, "Contraction mappings in b-metric spaces," Acta Mathematica Et Informatica Universitatis Ostraviensis, vol. 1, pp. 5-11, 1993.

[7] M. A. Alghmandi, N. Hussain, and P. Salimi, "Fixed point and coupled fixed point theorems on $b$-metric-like spaces," Journal of Inequalities and Applications, vol. 2013, p. 402, 2013.

[8] J. G. Heidary, A. Farajzadeh, M. Azhini, and F. Khojasteh, “A new common fixed point theorem for Suzuki type contractions via generalized y-simulation functions," Sahand Communications in Mathematical Analysis (SCMA), vol. 16, pp. 129-148, 2019.

[9] A. S. S. Alharbi, H. H. Alsulami, and E. Karapinar, "On the power of simulation and admissible functions in metric fixed point theory," Journal of Function Spaces, vol. 2017, 2017.

[10] B. Alqahtani, A. Fulga, and E. Karapınar, "Fixed point results on $\Delta$-symmetric quasi-metric space via simulation function with an application to ulam stability," Mathematics, vol. 6, no. 1, p. 208, 2018.

[11] H. Aydi, A. Felhi, E. Karapinar, and S. Sahmim, "A Nadlertype fixed point theorem in dislocated spaces and applications," Miskolc Mathematical Notes, vol. 19, no. 1, pp. 111-124, 2018.

[12] R. P. Agarwal and E. Karapinar, "Interpolative Rus-ReichCiric type contractions via simulation functions," Analele Universitatii "Ovidius"Constanta-Seria Matematica, vol. 27, no. 3, pp. 137-152, 2019.

[13] E. Ameer, H. Aydi, M. Arshad, and M. De la Sen, "Hybrid Cirić type graphic $\Upsilon, \Lambda$-contraction mappings with applications to electric circuit and fractional differential equations," Symmetry, vol. 12, no. 3, p. 467, 2020.

[14] E. Ameer, H. Aydi, M. Arshad, H. Alsamir, and M. Noorani, "Hybrid multivalued type contraction mappings in $\alpha \mathrm{k}$ complete partial b-metric spaces and applications," Symmetry, vol. 11, no. 1, p. 86, 2019. 
[15] N. Alamgir, Q. Kiran, H. Işık, and H. Aydi, "Fixed point results via a Hausdorff controlled type metric," Advances in Difference Equations, vol. 2020, no. 1, 2020.

[16] Z. Ma, A. Asif, H. Aydi, S. U. Khan, and M. Arshad, "Analysis of F-contractions in function weighted metric spaces with an application," Open Mathematics, vol. 18, no. 1, pp. 582-594, 2020.

[17] P. Patle, S. Patel, H. Aydi, and S. Radenović, "S. On $\mathrm{H}^{+}$-type multivalued contractions and applications in symmetric and probabilistic spaces," Mathematics, vol. 7, no. 2, p. 144, 2019.

[18] A. Felhi, H. Aydi, and D. Zhang, "Fixed points for $\alpha$-admissible contractive mappings via simulation functions," Journal of Nonlinear Sciences and Applications, vol. 9, no. 10, pp. 5544-5560, 2016.

[19] M. A. Alghamdi, S. Gulyaz-Ozyurt, and E. Karapınar, "A note on extended Z-contraction," Mathematics, vol. 8, no. 2, p. 195, 2020.

[20] V. Parvaneh, M. R. Haddadi, and H. Aydi, "On best proximity point results for some type of mappings," Journal of Function Spaces, vol. 2020, pp. 1-6, Article ID 6298138, 2020.

[21] K. Zoto, B. E. Rhoades, and S. Radenovic, "Common fixed point theorems for a class of $(s, q)$ - contractive mappings in $b$ - metric-like spaces and applications to integral equations," Mathematica Slovaca, vol. 69, no. .1, pp. 1-15, 2019.

[22] K. Zoto, B. E. Rhoades, and S. Radenovic, "Some generalizations for $(\alpha-\psi, \varphi)$ contractions in $b$ - metric-like spaces and application," Fixed Point Theory Application, vol. 2017, p. 26, 2017.

[23] K. Zoto, S. Radenović, and A. H. Ansari, "On some fixed point results for ( $\mathrm{s}, \mathrm{p}, \alpha)$-contractive mappings in b-metric-like spaces and applications to integral equations," Open Mathematics, vol. 16, no. 1, pp. 235-249, 2018.

[24] N. Hussain, S. Radenovi'c, and K. Zoto, "Common fixed point results of $(\alpha-\psi, \varphi)$-contractions for a pair of mappings and applications," Mathematics, vol. 6, no. 10, p. 182, 2018.

[25] K. Zoto, S. Radenovic, J. Dine, and I. Vardhami, "Fixed points of generalized $(\psi, s, \alpha)$-contractive mappings in dislocated and $b$ - dislocated metric spaces," Communications in Optimization Theory, vol. 2017, pp. 1-16, 2017.

[26] N. Fabiano, T. Dosenovic, D. Rakic, and S. Radenovic, "Some new results on $(s-q)$-Dass-Gupta-Jaggi type contractive mappings in $b$ - metric-like spaces," Filomat, vol. 2020, 2020.

[27] M. De la Sen, N. Nikolić, T. Došenović, M. Pavlović, and $\mathrm{S}$. Radenović, "Some results on ( $\mathrm{s}-\mathrm{q})$-graphic contraction mappings in b-metric-like spaces," Mathematics, vol. 7, no. 12, p. 1190, 2019.

[28] T. Abdeljawad, N. Mlaiki, H. Aydi, and N. Souayah, "Double controlled metric type spaces and some fixed point results," Mathematics, vol. 6, no. 12, p. 320, 2018.

[29] H. Afshari, H. Aydi, and E. Karapınar, "On generalized $\alpha$ - $\psi$-Geraghty contractions on b-metric spaces," Georgian Mathematical Journal, vol. 27, no. 1, pp. 9-21, 2020.

[30] S. Aleksic, Z. D. Mitrovic, and S. Radenovic, "Picard sequences in $b-$ metric spaces," Fixed Point Theory, vol. 21, no. 1 , pp. 35-46, 2020. 